\title{
Late diagnosis of total anomalous connection of pulmonary veins during the puerperium
}

\author{
$\underline{\text { Susan Pumacayo-Cárdenas }}{ }^{1 *}$
}

${ }^{1}$ Department of Cardiovascular Magnetic Resonance and Tomography, Fellowship, National Institute of Cardiology, Ignacio Chávez, México

\section{Corresponding Author: Susan Pumacayo-Cárdenas}

Address: Belisario Domínguez Sección 16, Belisario Domínguez Secc 16, Tlalpan, CDMX.

Received date: 25 June 2019; Accepted date: 4 July 2019; Published date: 11 July 2019

Citation: Pumacayo-Cárdenas S, "Late diagnosis of total anomalous connection of pulmonary veins during the puerperium”. Asp Biomed Clin Case Rep, vol.2, no.2: 4-8, 2019.

Copyright (C) 2019 Pumacayo-Cárdenas S, This is an open-access article distributed under the Creative Commons Attribution License, which permits unrestricted use, distribution, and reproduction in any medium, provided the original work is properly cited.

\begin{abstract}
The total anomalous pulmonary venous connection (TAPVC) is a rare congenital anomaly. We present a case of a 36-year-old woman, in the postpartum period during the physical examination there was a heart murmur and beating in hands and feet. Cardiac magnetic resonance reported supracardiac TAPVC and large atrial septal defect. The survival of these patients is very uncommon in adults and even worse during pregnancy.
\end{abstract}

\section{Keywords}

Anomalous Systemic Venous Drainage; Postpartum Period; Adult; Phase-Contrast MRI

\section{Introduction}

The total anomalous pulmonary venous connection (TAPVC) is a rare congenital anomaly, which corresponds to approximately $2 \%$ of all congenital heart defects $[1,2]$. The majority of patients present in the neonatal period with decompensated heart failure due to severe pulmonary hypertension or obstruction of pulmonary venous return $[3,4]$. The natural history of the disease shows that $20 \%$ survive until the first year of life without treatment [5]. Exceptionally, the symptoms may appear later in life or progressively, depending on some factors, which will be discussed later [6], the case we present is of a woman who is diagnosed with congenital heart disease during the puerperium. In some series, maternal mortality of patients with pulmonary hypertension, during pregnancy and the puerperium is very high.

Presentation of the Case
A 36-year-old female patient, from 13 years of age, began with dyspnea at great exertion and cyanosis of the lips. At 35 years of age, during his first pregnancy, he presented a slight increase in cyanosis in his lips, hands, and dyspnea at medium and large efforts, so he limited his physical activities [7]. She went to a health care center for the first time to attend her delivery, which was eutocic, without hemodynamic complications. The product was a newborn, full term, with adequate weight for gestational age, healthy. The hospital discharge was usually the second postpartum day. The findings in the physical examination were cyanosis, digital clubbing, without signs of respiratory distress (oxygen saturation 85\%, $\mathrm{FiO}_{2} 24 \%$ ), the hyperdynamic precordium, cardiac auscultation revealed a regular heart rhythm, a wide and fixed division of the second heart sound with accentuation of the pulmonary component and holosystolic murmur $4 / 6$ in the lung area irradiated to the neck. Liver palpable $4 \mathrm{~cm}$ below the costal margin. The peripheral pulses were symmetrical and there was no peripheral edema. The chest $\mathrm{x}$-ray revealed 
Citation: Pumacayo-Cárdenas S, "Late diagnosis of total anomalous connection of pulmonary veins during the puerperium”. Asp Biomed Clin Case Rep, vol.2, no.2: 4-8, 2019.

cardiomegaly and cardiac silhouette in the shape of a "snowman" (Fig-1A). Echocardiography was not optimal, found dilation of right cavities, atrial septal defect (ASD) of $15 \mathrm{~mm}$ and systolic pulmonary pressure of $70 \mathrm{mmHg}$. Twenty days postpartum, the patient comes to our institution, where the magnetic resonance imaging (MRI) shows the presence of total anomalous connection of pulmonary veins supracardiac variety. The confluence of the four pulmonary veins was wide (diameter 36x25 mm) and was connected to a dilated vertical vein, which in turn drained into a dilated innominate vein (at the mouth its diameter measured $32 \times 20 \mathrm{~mm}$ ), without evidence of obstruction (Fig-1B). Right atrium

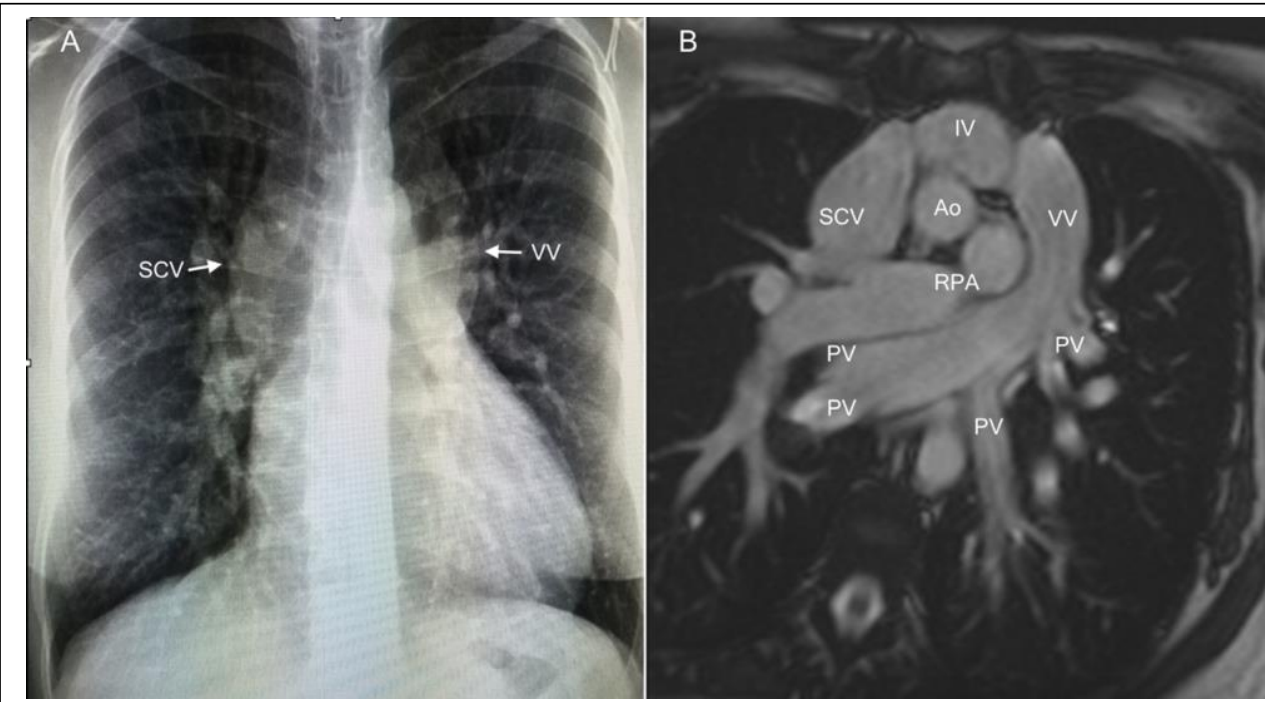

Fig-1: Chest X-ray

RPA (Right Pulmonary Artery)

A: Configuration of the heart and the superior mediastinal borders resembling a "snowman".

B: Echo gradient acquisition shows the pulmonary veins (PV) drain into an enlarged vertical vein (WV) which in turn drains into the innominate (IV) vein and then to the superior vena cava (arrow).

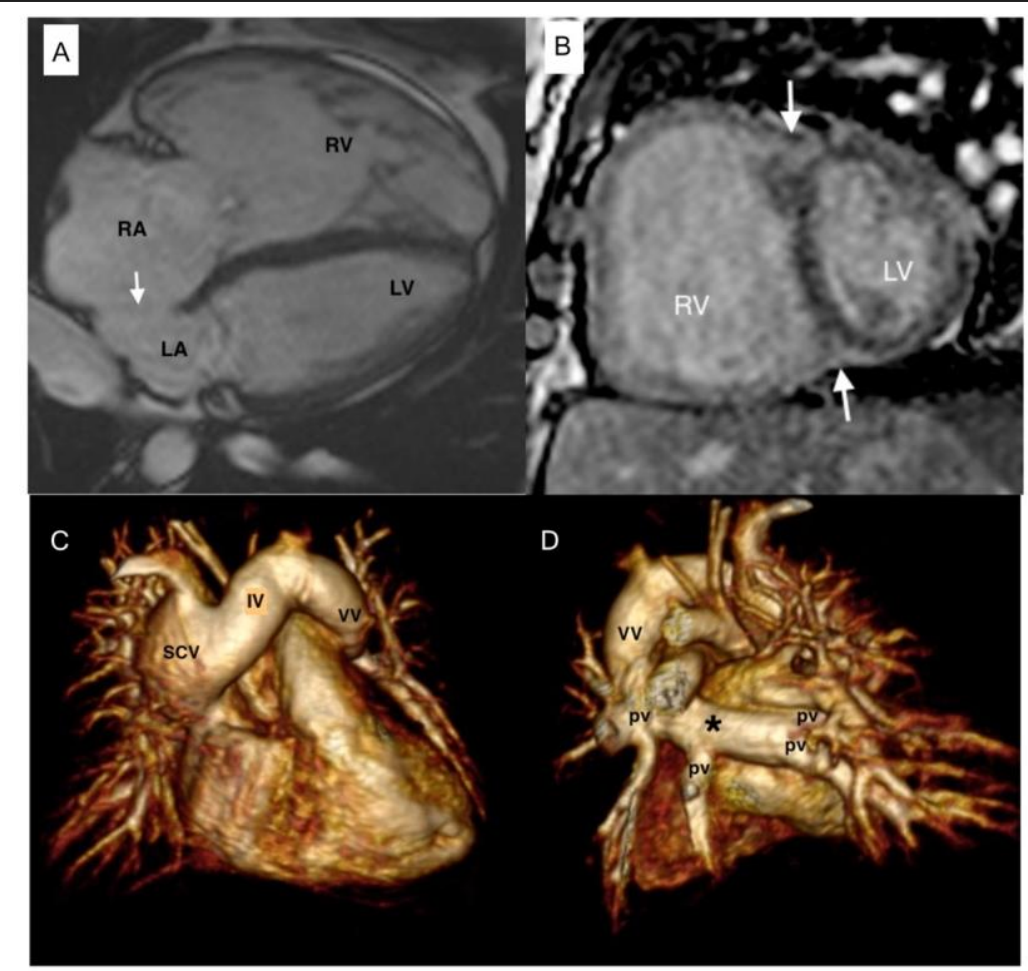

Fig-2: Cardiac Magnetic Resonance (CMR) 
Citation: Pumacayo-Cárdenas S, "Late diagnosis of total anomalous connection of pulmonary veins during the puerperium”. Asp Biomed Clin Case Rep, vol.2, no.2: 4-8, 2019.

VV (vertical vein); IV (innominate vein); RA (right atrium); SVC (superior vena cava)

A: Axial echo gradient acquisition shows four chamber view, showed that the right atrium and ventricle were significantly enlarged, the left atrium was small without pulmonary vein entrance. Note a large atrial septal defect (arrow).

B: Arrows point at zones of late gadolinium enhancement pattern of mid-wall fibrosis in sites of union of the left ventricle (LV) with the right and in the inferior wall of the $R V$.

C: The 3-D MRI image (anterior view) $y$

D: Posterior view, showed five pulmonary veins (pv) entered into a pulmonary venous sinus (*) connected through the vertical vein to innominate vein which entered into a significantly enlarged superior vena cava.

measured $23 \mathrm{~cm} 2 / \mathrm{m} 2$, left atrium $7.5 \mathrm{~cm} 2 / \mathrm{m} 2$. The ASD was superior venous sinus type of $25 \times 20 \mathrm{~mm}$, the short circuit from right to left and high pulmonary flow (Qp / Qs 2.9). Dilated right ventricle, systolic septal flattening, ejection fraction $48 \%$ and moderate tricuspid regurgitation; left ventricle with preserved ejection fraction. Trunk and branches of the pulmonary artery dilated. Presence of late gadolinium enhancement intramyocardial (Fig-2B).

Cardiac catheterization (Fig-3) reported pulmonary arterial hypertension, due to volume overload, even without the involvement of the pulmonary microvasculature (mean of the pulmonary pressure 6ommHg, pulmonary vascular resistance (PVR) 2.5 U Wood/m2, PVR: SVR 0.1). With these
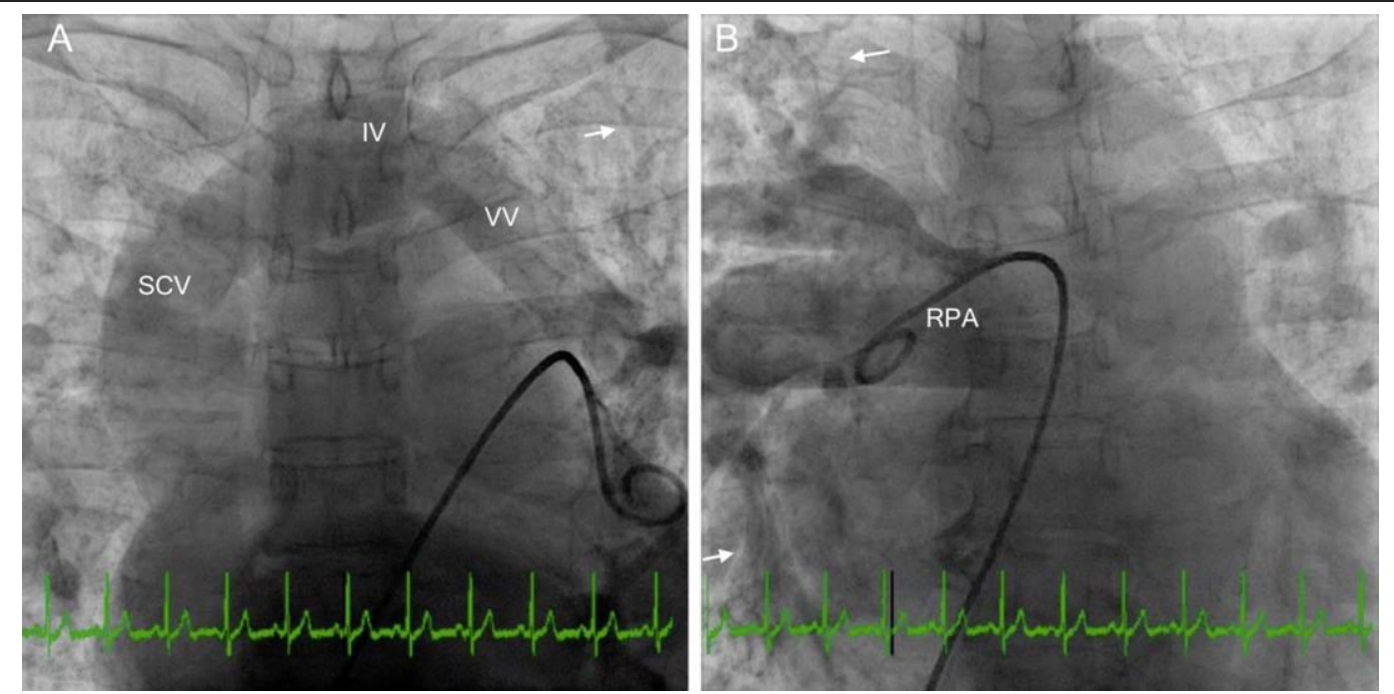

Fig-3: Cardiac Catheterization

VV (vertical vein); IV (innominate vein); SVC (superior vena cava)

A: Angiography in the left pulmonary artery (LPA), showed the absence of venous catheter obstruction (adequate contrast step in the drainage of the $V$ to the $I V$ vein and from this to the $S C V$.

B: Angiography in the right pulmonary artery (RPA), showed significant dilatation to the lobar and segmental branches, the distal vasculature is preserved (arrows).

hemodynamic parameters, it was decided to perform the respective total surgical repair.

Due to the large variations in anatomy and hemodynamics, TAPVC demonstrates a broad spectrum of clinical presentations ranging from the absence of symptoms to severe hypoxemia [8-11]. To survive to adulthood, there should be no obstruction between the pulmonary venous catheter, the systemic vein and the right atrium, an adequate size of the ASD, adequate oxygenation of the arterial blood and exclude the presence of severe pulmonary hypertension. Jian et al [11] in their study describes the average diameter of the pulmonary venous confluence $(25 \mathrm{~mm})$, the average size of the atrial septal defect (31mm) and the non-restrictive drainage site can provide conditions compatible with long-term survival term. In the present case, similar conditions were observed.

The thorough evaluation of these patients must include right cardiac catheterization, for the 
measurement of pulmonary pressure, PVR. Criteria for the closure of the short circuit have been proposed based on the baseline RVP $<4$ U Wood / m2ASC. Additional criteria are the type of defect, the age, the ratio PVR: SVR and the quotient Qp: Qs. There are no prospective data on the usefulness of the vasoreactivity tests, the closure tests or the lung biopsy for the evaluation of operability, however, its use will depend on the treating doctor [12].

In patients with $\mathrm{PH}$, characterized by having fixed and elevated RVP, there is great difficulty in managing high cardiac output during pregnancy and its increase during delivery, which leads to higher mortality. Clinical deterioration appears more frequently in the second trimester of pregnancy, corresponding to a $40 \%$ increase in cardiac output [12]. The diagnosis of this case is very rare in the adult, due to the high infant mortality when the corrective treatment is not carried out. The presence of a broad atrial septal defect, which allowed blood flow from right to left, the absence of obstruction of the venous system and the preservation of the pulmonary microvasculature prevented a fatal event during the development of this patient's life, in this way could be a candidate for corrective treatment.

\section{Conclusions}

The diagnosis of TAPVC is infrequent in adulthood. The case presented is exceptional, as it is an adult woman and in the postpartum period. Survival in these cases will depend on the absence of pulmonary venous obstruction, presence of large intracardiac septal defects that allow the right to left shunts. However, the presence of severe pulmonary hypertension and damage to the pulmonary vasculature is common and may limit the corrective treatment. MRI and catheterization are suitable diagnostic methods, for use in preoperative evaluation.

\section{Funding Information}

Self-financed

\section{Declaration of interests}

None

\section{References}

[1] S Sulaiman, GN Rajesh, "Anomalous left atrial drainage of the right superior caval vein with partial anomalous pulmonary venous return". Cardiology in the Young, vol.27, no.6: 1216-18, 2017.

[2] Ahmad N, Sell JE, "Closed technique for repair of right superior vena cava draining to left atrium”. Ann Thorac Surg, vol.55, no.6: 1560-70, 1993.

[3] Magalhães SP, Moreno N, Loureiro M, et al., "Anomalous pulmonary venous connection: an underestimated entity". Rev Port Cardiol, vol.35, no.12: 697-703, 2016.

[4] Craig JM, Darling RC, Rothney WB, "Total pulmonary venous drainage into the right side of the heart; report of 17 autopsied cases not associated with other major cardiovascular anomalies”. Lab Invest, vol.6, no.1: 44-64, 1957.

[5] Kim TH, Kim YM, Suh CH, et al., "Helical CT angiography and three-dimensional reconstruction of total anomalous pulmonary venous connections in neonates and infants". AJR, vol.175, no.5: 1381-86, 2000.

[6] Valsangia ER, Levasseur S, McCrindle BW, et al., "Contrast-enhanced MR Angiography of pulmonary venous abnormalities in children”. Pediatr Radiol, vol.33, no.2: 92-98, 2003.

[7] Avellana P, Segovia J, Lópe F, et al., "Hipertensión pulmonar y embarazo - Pulmonary hypertension and pregnancy”. Cardiocore, vol.47, no.4: 154-6o, 2012.

[8] Bharati S, Lev M, "Congenital anomalies of the pulmonary veins”. Cardiovasc Clin, vol.5, no.1: 23-41, 1973.

[9] Reddy KP, Nagarajan R, Rani U, et al., "Total anomalous pulmonary venous connection beyond infancy”. Asian Cardiovasc Thorac Ann, vol.19, no.3: 249-52, 2011.

[10] Jian XH, Huang J, Ding Y, et al., "Surgical Outcome of Isolated Total Anomalous Pulmonary Venous Connection in Adults: A 14-Year Experience”. J Card Surg, vol.27, no.6: 736-39, 2012.

[11] Atik FA, Jaramillo M, Afiune Y, et al., "Complete repair of total anomalous pulmonary venous connection in adult patient". Rev Bras Cir Cardiovasc, vol.24, no.1: 81-83, 2009.

[12] Galiè N, Humbert M, Vachiery JL, et al., "Guía ESC/ERS 2015 sobre diagnóstico y tratamiento de la hipertensión pulmonar". Rev Esp Cardiol, vol.69, 
Citation: Pumacayo-Cárdenas S, "Late diagnosis of total anomalous connection of pulmonary veins during the puerperium”. Asp Biomed Clin Case Rep, vol.2, no.2: 4-8, 2019.

Case Report

no.2: 177-259, 2016.

Keywords: Anomalous Systemic Venous Drainage; Postpartum Period; Adult; Phase-Contrast MRI 